\title{
Modeling, Mining, and Analyzing Semantic Trajectories: The Process to Extract Meaningful Behaviors of Moving Objects
}

\author{
Sana Chakri \\ FSTG, Cadi Ayyad University \\ Marrakesh, Morocco
}

\author{
Said Raghay \\ FSTG, Cadi Ayyad University \\ Marrakesh, Morocco
}

\author{
Salah El Hadaj \\ ENCG, Cadi Ayyad University \\ Marrakesh, Morocco
}

\begin{abstract}
Mobile devices leave a huge number of digital traces that are collected as trajectories, describing the movement of its users or a path followed by any moving object in geographical space over some period of time. However, those mobile devices provide just raw trajectories $(\mathrm{x}, \mathrm{y}, \mathrm{t})$, ignoring information about their related contextual data, these additional data contribute in producing significant knowledge about movements and provide applications with richer and more meaningful knowledge. Therefore, researchers focus on transforming raw trajectories into semantic trajectories by combining the raw mobility tracks with related contextual data and creating a new type of trajectories called "semantic trajectories", then applying mining techniques. This paper study closely the current researches on modeling and mining semantic trajectories so far, and try to investigate by proposing a descriptive schema including all steps that users can browse from the construction of the trajectories to the analyze of behaviors extracted.
\end{abstract}

\section{Keywords}

Semantic trajectories, extracting knowledge, semantic enrichment, spatial data mining.

\section{INTRODUCTION}

Trajectory data play a fundamental role to a huge number of applications, such as transportation management, urban planning, tourism and animal migration. This type of data is normally obtained from mobile devices that capture the position of an object and his time interval, and it is available for use in the format of raw trajectories. Several data mining methods for raw trajectories have been proposed in the last few years. These approaches are characterized by focusing on the geometrical properties of trajectories, without considering any contextual data. As consequence, these methods tend to discover geometric patterns (based usually on the concept of dense regions or trajectory similarity), which may give less meaningful information, since the data themselves are very limited and have no direct link to other information. Semantic trajectories, however, have more meaningful data; it is a growing trend that has recently emerged in geographic information science and spatiotemporal knowledge discovery. Their patterns are independent of spatial coordinates, and can be located in sparse regions and may not have geometric similarity, from now on semantics plays an essential role in several applications, it is becoming an important research issue in GIS (Geographic Information Systems), however, Complex technical operations and data functions are necessary to add semantics to trajectories in order to facilitate their analysis and knowledge extraction from the user's point of view [1]. In this paper we discuss the existing literature on semantic trajectories modeling and mining and propose a new descriptive schema for the researches done on this field, the proposed schema will classify minutely the semantic trajectories process (from construction to behavior extraction and analysis) through three main step: the first one is raw trajectory modeling where the trajectory will be constructed and structured, the second one is semantic trajectory modeling were the trajectory will be enriched (segmented and annotated) for eventual use. And the last one is semantic trajectory mining where the trajectory will be used according to given application domain. Several similar efforts were done

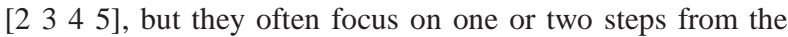
process, whether it was the mining step, enrichment, preprocessing, or post-processing. This paper extends their works by covering the whole process and giving a descriptive schema which classify the trajectory enrichment process from reconstruction to analyzing.

The rest of the paper is organized as follows: Section 2 present the schema of semantic trajectory process. Next we discuss the three steps of the schema proposed: raw trajectory modeling (section 3), semantic trajectory modeling (section 4) and semantic trajectory mining (section 5), section 6 gives an application example to demonstrate the effectiveness of the process schema and section 7 concludes our conducted study.

\section{SCHEMA OF SEMANTIC TRAJECTORY PROCESS}

In the current research on semantic trajectories, researchers go through many steps before their data will be extracted and analyzed, we can say that the whole process can be structured on three basic phases that are important and fundamental to prepare trajectories for eventual use. The first phase is raw trajectories modeling, where the important task is the structure of the trajectory, in this phase users usually try to prepare trajectories by cleaning the GPS feeds and structuring the parts of trajectories that will be stored and exploited in order to make healthy raw trajectory. Well The second phase takes as input these raw trajectories, and tries to segment it into episodes of stops and moves and annotated it with related contextual data. In order to product semantic trajectories, these semantic trajectories will be the input of the third phase which is semantic trajectory mining, where the user will be able to classify, cluster and eventually apply data mining algorithms to extract behavior knowledge depending on its application field.

Figure 1 presents the schema of semantic trajectory process proposed. 


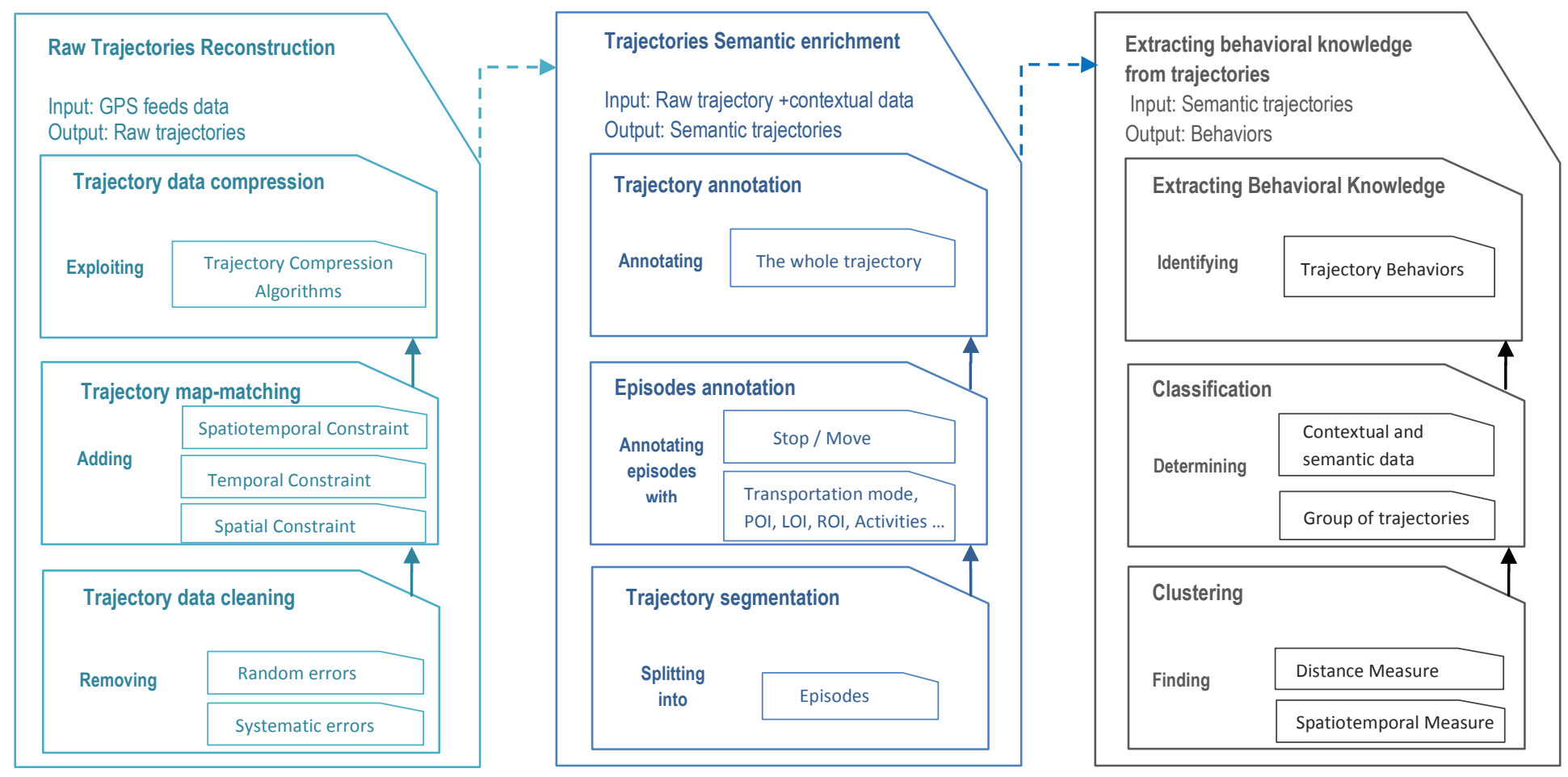

Figure 1 : Semantic trajectory process

\section{RAW TRAJECTORY MODELING}

Raw data modeling is the first task of semantic trajectory process. It is the part of constructing and structuring data to support the enrichment of trajectories [6 7]. In this phase there are three main levels that evolve as we progress from the initial requirements to the construction of healthy trajectories; this section presents these three levels: (1) Data cleaning, (2) Map-matching, (3) Trajectory compression. In which the global aim is to turn the imperfect raw movement data into a trajectory dataset that is manageable. In other words we try to construct trajectories that are: clean with minimal noise possible, map-matched and compressed as much as possible. Notice that the user can skip tasks without going through all the three steps, actually we try to discuss the full process that user can browse to have raw trajectories but the user can be satisfied with one or two tasks as needed.

\subsection{Trajectory data cleaning}

Trajectory data cleaning, also called data cleansing or scrubbing, deals with detecting and removing errors and inconsistencies from trajectory data in order to improve the quality of the trajectory data. In this first step, the main idea is to clean dataset collected by mobile sensors which are sometimes imprecise due to several types of noise caused by the limitations of positioning systems [8], or about the user's privacy protection. According to [2] there are two types of errors: systematic errors caused by systems limitation and random errors caused by the satellite orbit. In general errors refer to the spatial positions, while the temporal positioning is often more exact [9].

Notice that the cleaning techniques cannot guarantee completely removing errors for every GPS point, but it tends to the objective of data cleaning which is to minimize the total number of errors for all GPS records.

\subsection{Trajectory Map-Matching}

The trajectory cleaning methods discussed previously are designed for objects moving freely and without any constraint. However, in many applications moving objects are restricted to move within a given network. For instance the car in Figure 2 cannot move across the buildings to arrive at the final destination, it needs to follow the road network, this type of constraints is spatial. Trajectories often meet with spatial, temporal, or spatiotemporal type of constraints.

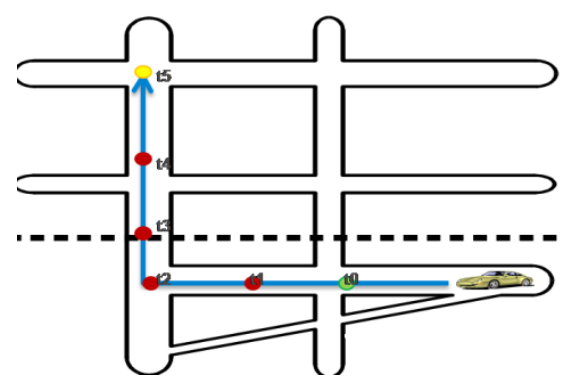

Figure 2: Car trajectory with spatial constraint

Map matching is applied for transforming the raw trajectories into map-matched trajectories[10], this manipulation may be realized either in the data cleaning step, by replacing trajectory positions by points on the network that are the most likely position of the moving object, or directly in the enrichment step. Map-matching can be performed either Online (the algorithm should be fast as new positions flow in real time). Or offline (the algorithm is run after the acquisition of all positions).

\subsection{Trajectory data compression}

Trajectory data grow gradually and intensively as the time goes by, this is due primarily to the large availability of mobile sensors, such as GPS-enabled smart phones. Accordingly, there are a huge number of data stored in 
database, thereby the necessity of trajectory data compression which plays an essential task of trajectory reconstruction. The objectives of trajectory compression are to reduce the size of the dataset, and to allow computations with low complexity, and also to support low deviation between the reduced trajectory and the original one, without warping the trend of the trajectory or distorting data [11].

\section{SEMANTIC TRAJECTORY MODELING}

After constructing raw data, and to prepare trajectories for data mining, the mid step is to add semantics to these trajectories, adding semantics is achieved by linking the spatiotemporal units with semantic knowledge from the geographic data and application domain data, in order to facilitate the user's task to analyze and interpret the knowledge in mining steps. In this section we will discuss the trajectory semantic enrichment process, which is based on segmenting trajectory into sub-trajectories called episodes then annotating them for more enrichment.

\subsection{Segmentation}

A way of segmenting a trajectory is to split its path into periods of time when the object is considered as constant (stops), and periods where the object is moving (moves). the first data model looking at the trajectories from this point of view is Stop/move model[12 13], according to this model, stops are the important places of a trajectory where the moving object has remained for a period of time, while moves are the sub-trajectories between stops. Thus, semantic trajectory: is a sequence of alternating stops and move episodes

Finding Stops is one of the most important challenges to segment trajectories, for this, different approaches have been proposed: the first one is the velocity-based approach, where the GPS point belongs to a stop or a move episode according to a speed threshold. When this approach is not enough for determining stops the users can add the Density-Based approach that It consider besides the speed, the maximum diameter that the moving object has traveled during a given time duration.

Another important approach is identifying stops by using a combination of raw data, contextual geographic information, and application information; this is done by identifying which places are of interest to the specific application (POI), the first algorithm that verify this constraint is SMoT method, according to this algorithm a stop is a position where a trajectory holds on for minimal time duration and that intersect with the position of one of the POIs (point of interest) defined by the application. Afterward, other extensions of SMoT have been developed. We mention IBSMoT (Intersection-based) [14], interesting for applications like tourism and urban planning. CB-SMoT (Speed-based clustering) [15], interesting for applications where the speed is important like traffic management. DB-SMOT (Directionbased clustering) [16], interesting in applications where the direction variation is important like fishing activities.

Notice that trajectories can be segmented with other criteria like the means of transportation used by the moving object, $[17$ 18]; for instance this knowledge is useful in transportation management.

\subsection{Annotation}

After segmenting trajectories, the segmented episodes need to be annotated in order to be more enriched and to give meaning to the semantic trajectories, Usually episodes are annotated with their defining annotation: for instance, stop and move episodes are annotated as "stop" or "move", transportation mode episodes are annotated as "walk", "car", etc [19 20]. However episodes may have more than one annotation to achieve application requirements, for instance episodes may be additionally annotated with the corresponding POI, LOI or ROI where the moving object stopped. Its type (e.g., home, work-place, restaurant), or even their pursued activities during the stop and move.

Roughly, Very few researches had focused on annotating moves, while there is an important part of research for understanding stops [21 22] because stopping generally means that there is something of interest to do there.

\section{SEMANTIC TRAJECTORY MINING}

In every application domain, using contextual and semantic information enriches the meaning of the extracted information, it can helps to understand the behaviors and May reveal behaviors that would be difficult to identify without using semantics. Knowledge discovery from trajectories aims essentially at identifying behaviors in two categories: unknown behaviors or specific behaviors [23 24]. Techniques used to identify behaviors are firstly clustering trajectories, then classifying trajectories into predefined classes (fishing ship trajectories), discovering common sequences of movements (going from home to work then to shopping center) and identifying trajectories of objects whose movement is somehow related to each other, in particular, objects moving together (flocks of animals).

\subsection{Semantic trajectory knowledge discovery}

For knowledge discovery in semantic trajectories, the integration with contextual geographic information plays an essential role towards the discovery of readily understandable behaviors. Considering the semantics of space and time and some contextual data, it gives more meaning to a behavior; otherwise it is important to observe that the integration of trajectories with contextual and semantic, spatial and temporal information is vital for the discovery of meaningful behaviors, because the majority of behaviors extracted from semantic trajectories cannot be obtained from raw data only. Two machine learning techniques of discovery of behaviors which based on similarity evaluations are popular in this context: clustering and classification.

\subsubsection{Clustering}

Is an unsupervised learning method that tries to group a set of objects and find whether there is some relationship between the objects, it is applied to determine groups of trajectories from a large database analysis. The groups are computed based on their similarities or dissimilarities between the whole trajectories or along some subparts of the trajectories. The computation of similarity is based on a distance measure [25], the usual distance measures are spatial distance and temporal distance but we can have similarities based on contextual data too. Trajectory clustering has been used in several contexts such as trajectory searching and querying [26], trajectory visualization [27] and processing of trajectory uncertainty [28].

\subsubsection{Classification}

After clustering the semantic trajectories to find the interesting segments, users can define a class for each cluster and apply classification to produce the classes by using a complementary supervised method called trajectory 
classification which aims to differentiate between trajectories or its segments of different status by Tagging the whole trajectory or its segment with semantic labels. Techniques most used in literature are: non-parametric (e.g. K nearest neighbor), mathematical models (e.g. neural networks), and rule based models (e.g. decision trees).

\subsection{Extracting behavioral knowledge}

Once the trajectories are built, enriched, well clustered and eventually classified, users at this level can apply data mining to extract meaningful knowledge and analyze behaviors of their moving objects, they can specify different granularity levels according to its needs, there is two types of granularities that user can manage: spatial and temporal, depending on the application, the time granularity can change into year, month, week, day, hour, etc. the same goes for the space granularity, figure 3 show an example of different granularities level that we can have.

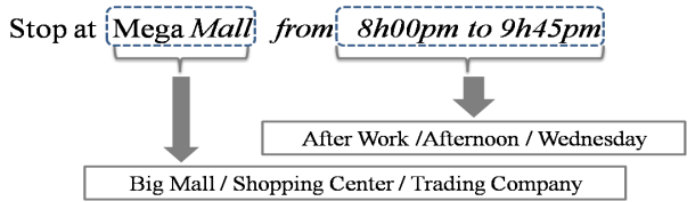

Figure 3 : STOPS at Multiple-Granularities

If we use semantic trajectories with stops annotated with POIs (Big Mall/ Shopping Center/ Business Company) as shown in Figure 3, we can discover the meaning of the semantic behaviors: stop at Mega Mall, stop at shopping center, or stop at Trading Company, so for the same data we can have a lot of meaning according to the application point of view. The figure present also three levels of temporal granularities that can be useful for different cases of study.

Researches leads to several kinds of semantic behaviors that can be mined by using semantic trajectories, the most popular is the sequential pattern (visiting sequences of places), the frequent Pattern (visiting the same places with a certain frequency), the associated pattern (visiting associations of places for instance trajectories that visit hotels do also visit touristic places), the outlier pattern (trajectories that have different behavior in relation to other trajectories), etc.

\section{APPLICATION EXAMPLE AND PROCESS OVERVIEW}

Hereafter we present example of an application studying the behavior of citizens about their dally activities on shopping centers, considering the trajectories of citizens in Marrakesh city, for that we implement an overview of the schema proposed using the data mining toolkit Weka-STPM as software Architecture for semantic trajectory data mining and visualization [29 30], and the PostGIS as database. Notice that there are other data mining toolkits like MoveMine [31], this toolkit provides a user interface where he can select a data set and the corresponding raw data is plotted on the Google Map, this toolkit can interact with Google Earth for 3D visualization.

Let's consider a dataset of citizens GPS feeds represented as sample points (figure 4). The first step is modeling raw trajectory, in this step we construct a healthy raw trajectories from GPS feed, in our case study we use cleaning and mapmatching process to minimize the noise and to have a sound set of trajectories, we don't need to compress our trajectory because we suppose that GPS feeds are regularly captured over some period of time, for this step, table 1 and figure 5 present a part of our raw trajectories after construction process.

$\begin{array}{llll}\text { type } & \text { time latitude } & \text { longitude } & \text { name desc } \\ \text { T } & 2010-07-0520: 00: 00 & 31.640677245 & -8.000024201 \\ \text { T } & 2010-07-0520: 00: 10 & 31.637291074 & -7.999375828 \\ \text { T } & 2010-07-0520: 00: 20 & 31.637290944 & -7.999496521 \\ \text { T } & 2010-07-0520: 00: 30 & 31.635883714 & -8.000471662 \\ \text { T } & 2010-07-0520: 00: 40 & 31.636611209 & -8.001259065 \\ \text { T } & 2010-07-0520: 00: 50 & 31.637008548 & -8.001922502\end{array}$

Figure 4: Part of citizens GPS feeds

The part of citizens GPS feeds must contain essentially sequence of latitude/longitude position coordinates and the time when each position is captured since those two types of data present the important attributes to transform raw trajectories into semantic ones.

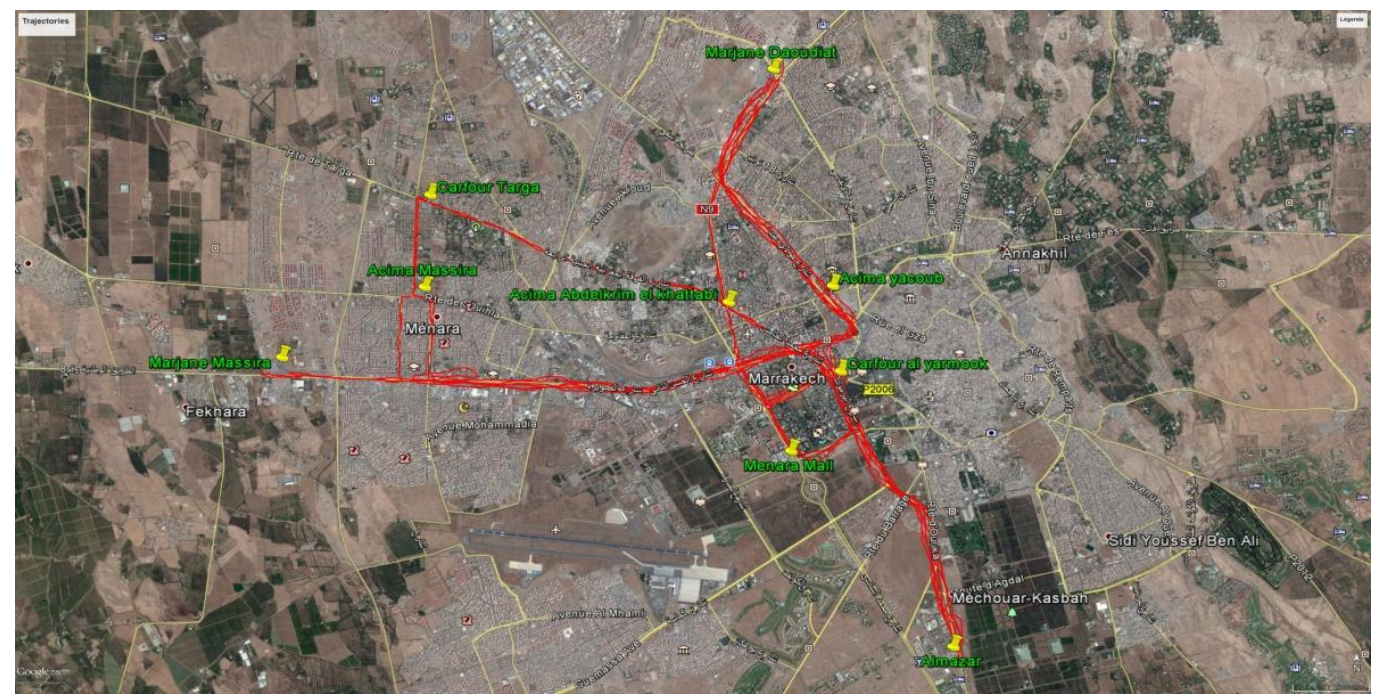

Figure 5: Citizens raw trajectories 
Table 1: Part of raw trajectory dataset

\begin{tabular}{|c|c|c|c|}
\hline Tid & Geometry & Timestamp & $\ldots$ \\
\hline 1 & $\begin{array}{c}\text { POINT(31.640677245 - } \\
8.000024201)\end{array}$ & 20:00:00 & \\
\hline 1 & $\begin{array}{c}\text { POINT(31.637291074 - } \\
7.999375828)\end{array}$ & $20: 00: 10$ & \\
\hline 1 & $\begin{array}{c}\text { POINT(31.637290944 - } \\
7.999496521)\end{array}$ & 20:00:20 & \\
\hline$\ldots$. & ... & & \\
\hline 2 & $\begin{array}{c}\text { POINT(31.640871258 - } \\
8.005873786)\end{array}$ & 21:00:00 & \\
\hline
\end{tabular}

Besides the trajectory data of citizens, there is a need of geographic information of the city. So after configuring and cleaning trajectory datasets, now we can add semantics to trajectories. Thereafter, we will exploit the semantic trajectory modeling and mining steps, First the raw trajectories will be segmented and annotated then clustered, we do that by using the concept of stops and moves and the algorithm IB-SMoT, Notice that the segmentation and annotation of trajectories in the toolkit Weka-STPM are provided at the same time when we enrich the trajectories in order to facilitate the procedure to users. To enrich our trajectories we need some geographic data, so besides the trajectory data of citizens, there is also contextual data of the city. Let us suppose that for this application, the interesting feature types (candidate stops) include all types of Markets that exist in Marrakesh city. Table 2 presents a part of candidate stops which represent the contextual data for our application domain.

Table 1 : Shopping areas (candidate Stops)

\begin{tabular}{|c|c|l|c|}
\hline Gid & Type & Name & Geometry \\
& & & \\
\hline 1 & T_Company & Menara & POINT(31.6185 \\
& & Mall & $-8.009474)$ \\
\hline 3 & H_Market & Marjane D & POINT(31.6674 \\
& & & $51,-8.012319)$ \\
\hline 5 & S_Market & Acima G & POINT(31.6370 \\
& & & $82,-8.018024)$ \\
\hline
\end{tabular}

Merging raw trajectories with contextual data lead to produce semantic trajectories in two relations Stop and Move. Stops and moves can be computed on the spatial granularity of instance or type depending of the application needs; in this experiment we tend to compute stops on granularities of type since that what interested us is the type of the store frequenting by citizens, not essentially its name. Table 3 shows a part of a stop dataset with spatial granularities of type after applying the algorithm IB-SMoT which merges between raw trajectories and candidate stops already existed in the dataset.

Table 2: Part of semantic trajectories - Stop dataset

\begin{tabular}{|c|c|l|l|c|c|}
\hline Sid & Tid & Gid & StopType & Startime & EndTime \\
\hline 1 & 1 & 1 & T_Company & $20: 00: 00$ & $22: 00: 00$ \\
\hline 2 & 1 & 3 & H_Market & $15: 00: 00$ & $17: 00: 00$ \\
\hline 3 & 2 & 5 & S_Market & $15: 00: 00$ & $20: 30: 00$ \\
\hline
\end{tabular}

Once the stops and moves are generated, which is the phase when semantics are added to trajectories and when time is most consumed between all steps of the knowledge discovery process, the data are ready for multiple-level mining. Furthermore, we specify what should be considered in the mining step and manage the aggregation in higher granularity levels (generalization) in order to apply mining algorithms for generating several types of patterns. Notice that generating stops and moves from trajectories is normally realized for once, while data mining algorithms may be applied to these data several times until extracting meaningful patterns.

According to the application domain and the user objectives, different data mining algorithms can be applied to the dataset of stops and moves; the most important are frequent patterns, association rules, and sequential patterns. For the rest of this experimental study we lead to extract some frequent patterns to analyze citizens' behavior regarding to different types of markets that exist in the city of Marrakesh.

After adding semantics to trajectories with the method CBSMoT, we transformed the data into different space and time granularities and then applied the algorithm for generating frequent patterns; at this level we can use the semantic trajectory data mining query language (ST-DMQL) [32] to specify semantic enrichment of trajectories with contextual domain information. Figure 6 shows the result of frequent pattern mining process on semantic trajectories considering two different granularities for the time dimension: weekdays and weekends.

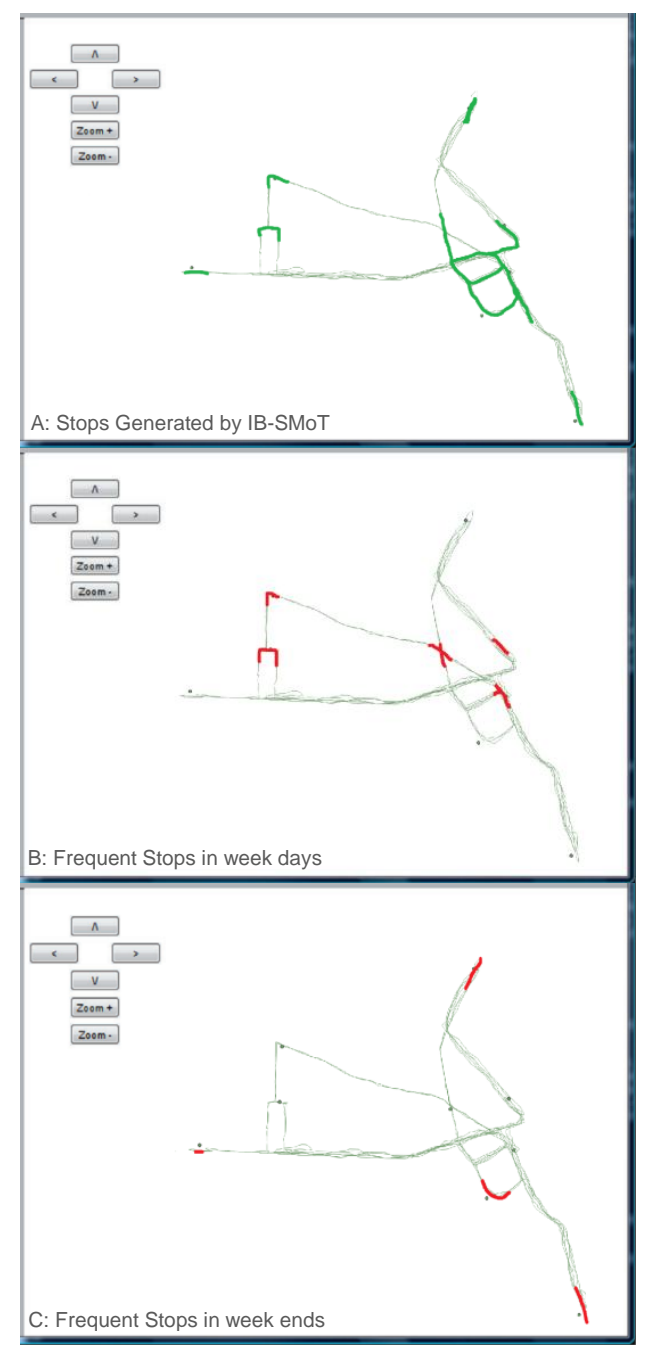

Figure 6: Patterns extracted from semantic trajectories 
In this figure we have three results: (A) represent all stops computed by IB-SMoT, (B) shows stops computed in weekdays, these stops are located essentially near to small and medium types of Market. And (C) shows stops computed in weekends which report generally, besides than super Markets to hyper Markets and Malls.

The information extracted by this pattern show that Marrakesh citizens frequent the small stores more than big surfaces, besides those who frequent big surfaces spend more time than those visiting super markets or small department stores, otherwise there is a relationship between the size of the building, and how long people attending it and spend time in it.

Finally we can say that Behaviors extracted from semantic trajectories cannot be obtained from raw data only. According to the kind of knowledge the user is interested in, different data mining algorithms can be applied to the dataset of stops and moves. And without using such kind of conceptualization it would be hard to understand moving behavior in trajectories.

\section{CONCLUSION}

In this paper we have shown that trajectory knowledge discovery depends directly on the application domain, and therefore there is a need to integrate geographic information into trajectories in order to create semantic trajectories before extracting meaningful patterns. We have presented for that a descriptive schema to explain the semantic trajectory knowledge process, in which we present the main steps that ensure complete acquisition of semantic trajectories in order to extract realistic behaviors according the application point of view, from now on user will spend less time to understand the patterns, since the resultant semantic trajectories are more summarized and interpreted.

The proposed schema process is general enough to cover different application domains; we have been proposed an experimental use case to reveal its effectiveness. The future ongoing work is the study and the exploitation of the clustering techniques to find unknown stops that can reveal more important knowledge.

\section{ACKNOWLEDGMENT}

I would like to thank my two supervisors for their commitments and guidelines as well as their mental support during the preparation of this work.

\section{REFERENCES}

[1] Van Hage W R, Wielemaker J, and Schreiber G 2010 The space package: Tight integration between space and semantics. Transactions in GIS 14: 131-46

[2] Parent C, Pelekis N, Theodoridis Y, Yan Z, Spaccapietra S, Renso C, Andrienko G, Andrienko N, Bogorny V, Damiani ML, Gkoulalas-Divanis A, Macedo J (2013) Semantic trajectories modeling and analysis. ACM Comput Surv 45:1-32

[3] 8. Yan Z, Spaccapietra S (2009). Towards semantic trajectory data analysis: a conceptual and computational approach. VLDB PhD Work 15(2):165- 190

[4] Luis Otavio Alvares , Vania Bogorny, Bart Kuijpers, Bart Moelans, Jose Antonio Fernandes de Macedo, Andrey Tietbohl Palma. Towards Semantic Trajectory
Knowledge Discovery. Databases and Theoretical Computer Science 2007

[5] Basma H. Albanna, Ibrahim F. Moawad, Sherin M. Moussa, Mahmoud A. Sakr. Semantic Trajectories: A Survey from Modeling to Application. Information Fusion and Geographic Information Systems (IF\&GIS' 2015) Lecture Notes in Geoinformation and Cartography 2015, pp 59-76

[6] Gudmundsson, J. Vankrevled, M. and Speckmann, B. 2007. Efficient detection of patterns in $2 \mathrm{~d}$ trajectories of moving points. Geoinformatica 11, 195-215

[7] Benkert, M., Gudmundsson, J., Hubner, F., and Wolle, T. 2008. Reporting flock patterns. Comp. Geom. 41, $111-125$.

[8] H. Fan, Q. Wu and Y. Lin. Behavior-Based Cleaning for Unreliable RFID Data Sets. Sensors, 2012, 12: 1019610207.

[9] Jun, J, Guensler, R., and Ogle, J. 2006. Smoothing methods to minimize impact of global positioning system random error on travel distance, speed, and acceleration profile estimates. Transport. Res. Rec. J. Transport. Res. Board 1972, 1, 141-150.

[10] Quddus, M. A., Ochieng, W. Y., and Noland, R. B. 2007. Current map-matching algorithms for transport applications: State-of-the art and future research directions. Transport. Res. Part C: Emerging Technol. $15,5,312-328$

[11] Schmid, F., Richter, K.-F., and Laube, P. 2009. Semantic trajectory compression. In Proceedings of the 11th International Symposium on Advances in Spatial and Temporal Databases. 411-416.

[12] Spaccapietra, S., Parent, C., Damiani, M. L., Macedo, J. A., Porto, F., and Vangenot, C. 2008. A conceptual view on trajectories. Data Knowl. Engin. 65, 126-146

[13] Alvares, L. O., Bogorny, V., Kuijpers, B., Demacedo, J. A. F., Moelans, B., and Vaisman, A. 2007. A model for enriching trajectories with semantic geographical information. In Proceedings of the 15th ACM International Symposium on Advances in Geographic Information Systems (ACM-GIS'07). 162-169, ACM Press, New York.

[14] Palma, A. T; Bogorny, V.; Kuijpers, B.; Alvares, L.O. A Clustering-based Approach for Discovering Interesting Places in Trajectories. In: 23rd Annual Symposium on Applied Computing, (ACM-SAC'08), Fortaleza, Ceara, 16-20 March (2008) Brazil. pp. 863-868

[15] Nanni, M., Pedreschi, D. 2006. Time-focused clustering of trajectories of moving objects. Journal of Intelligent Information Systems 27(3) (2006) 267-289

[16] Manso, J. A. ; Times, V. C. ; Oliveira, G. ; Alvares, Luis Otavio ; Bogorny, V. DB-SMoT: A Direction-Based Spatio-Temporal Clustering Method. In: IEEE International Conference on Intelligent Systems (IS), 2010, Londres. Proceedings of the IEEE International Conference on Intelligent Systems.

[17] Z., Chakraborty, D., Parent, C., Spaccapietra, S., and Aberer, K. 2011. SeMiTri: A framework for semantic annotation of heterogeneous trajectories. In Proceedings 
of the 14th International Conference on Extending Database Technology (EDBT'11). 259-270

[18] Zheng Y, Chen Y, Li Q, Xie X, Ma W-Y (2010)

[19] Understanding transportation modes based on GPS data for web applications. ACM Trans Web 4(1)

[20] Yan Z, Spremic L, Chakraborty D, Parent C, Spaccapietra S, Aberer K (2010) Automatic construction and multi-level visualization of semantic trajectories. In: Proceedings of the $18^{\text {th }}$ SIGSPATIAL international conference on advances in geographic information systems-GIS ' 10, vol 12, p 524.

[21] Xiu-Li Z, Wei-Xiang X (2009) A clustering-based approach for discovering interesting places in a single trajectory. In: 2009 second international conference on intelligent computation technology and automation, pp 429-432

[22] Moreno B, Times V, Renso C, Bogorny V (2010) Looking inside the stops of trajectories of moving objects. In: Geoinfo

[23] Zhixian Y (2011) Semantic trajectories: computing and understanding mobility data In: Ph.D. dissertation, Swiss Federal Institute of Technology, Information and Communication Dept., Lausanne

[24] Ying JJ-C, Lee W-C, Tseng VS (2013) Mining geographic-temporal-semantic patterns in trajectories for location prediction. ACM Trans Intell Syst Techno 1 5

[25] Ilarri S, Stojanovic D, Ray C (2015) Semantic management of moving objects: a vision towards smart mobility. Expert Syst Appl 42(3):1418- 1435
[26] Han, B., LIU, L., and Omiecinski, E. 2012. NEAT: Road network aware trajectory clustering. In Proceedings of the 32nd International Conference on Distributed Computing Systems (ICDS'12). 142-151.

[27] Panagiotakis, C., Pelekis, N., Kopanakis, I., Ramasso, E., and Theodoridis, Y. 2012. Segmentation and sampling of moving object trajectories based on representativeness. IEEE Trans. Knowl. Data Engin 24, 7, 1328- 1343.

[28] Rinvizillo, S., Pedreschi, D., nanni, M., Giannotti, F., Aandrieko, N., and Andrieko, G. 2008. Visually-driven analysis of movement data by progressive clustering. Inf. Vis. 7, 3-4, 225-239.

[29] Pelekis, N., Kopanakis, I., Kotsifakos, E. E., Frentzos, E., and Theodoridis, Y. 2011. Clustering uncertain trajectories. Knowl. Inf. Syst. 28, 1, 117-147.

[30] Alvares L, Palma A, Oliveira G, Bogorny V (2010) Weka-STPM: from trajectory samples to semantic trajectories. In: Proceedings of the Workshop Open Source Code, vol 1.

[31] V Bogorny, H Avancini, B C de Paula, C R Kuplich and L O Alvares. Weka-STPM: a Software Architecture and Prototype for Semantic Trajectory Data Mining and Visualization Transactions in GIS, 2011, 15(2): 227-248

[32] Li Z, Ji M, Lee J, Tang L, Yu Y, Han J, Kays R (2010) MoveMine. In: Proceedings of the 2010 international conference on management of data-SIGMOD ' $10, \mathrm{p}$ 1203

[33] Bogorny V, Kuijpers B, and Alvares L O 2009 St-dmql: A semantic trajectory data mining query language. International Journal of Geographical Information Science 23: 1245-76 